Implementation and Effects of an Information TechnologyBased

Intervention to Support Speech and Language Therapy Among Stroke Patients with Aphasia: Protocol for a Virtual Randomized Controlled Trial

\title{
VoiceAdapt App Screenshots
}

Esther S. Kim, PhD, Laura Laird, BA, Carlee Wilson, MSc, Till Bieg, MSc, Philip Mildner, PhD, Sebastian Möller, PhD, Raimund Schatz, PhD, Mag. Stephanie Schwarz, Robert Spang, MSc, Jan-Niklas Voigt-Antons, PhD \& Elizabeth Rochon, PhD 


\section{Login page}

\section{Welcome to VoiceAdapt!}

Please log in to begin the exercises.

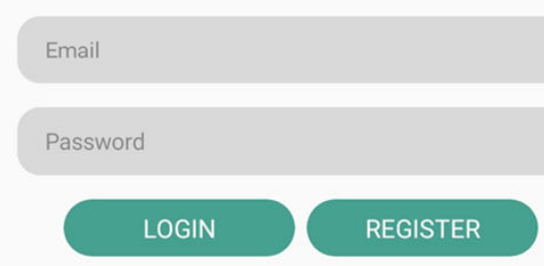




\section{Registration: User profile}

Here the user fills out profile information that is used to generate training content personalized to their interests.

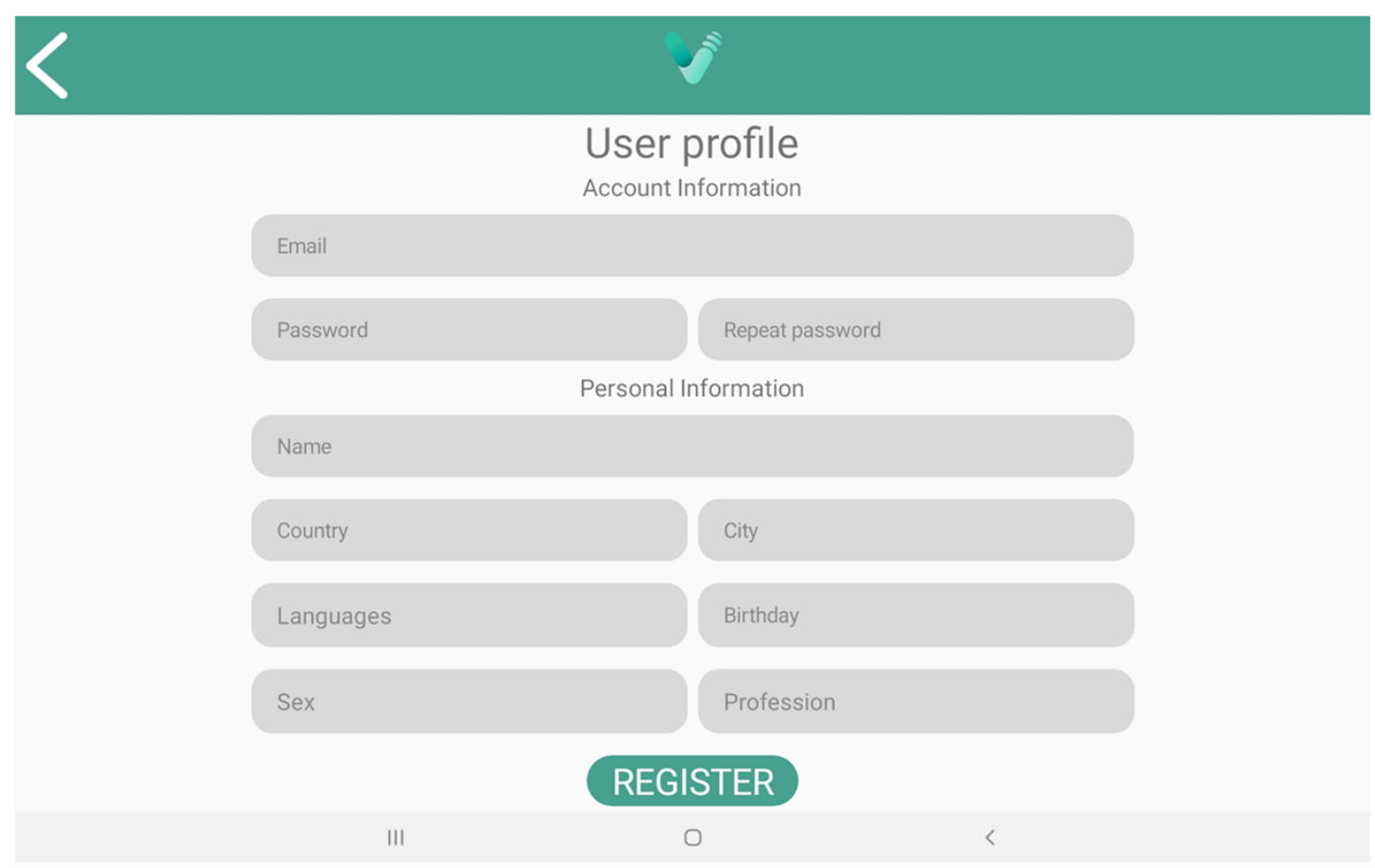




\section{Start page}

The therapist sets the weekly goal (60 words/week for participants enrolled in the trial). The progress bar shows weekly progress.

The app alternates between training items using Semantic Feature Analysis (SFA) and Phonological Components Analysis (PCA) each week.

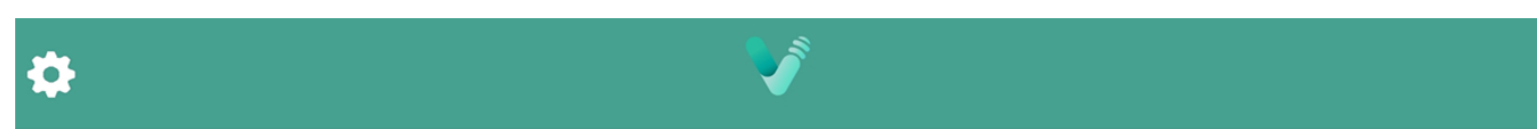

\section{QHello carlee!}

Would you like to practice right away?

Weekly goal : 2 words

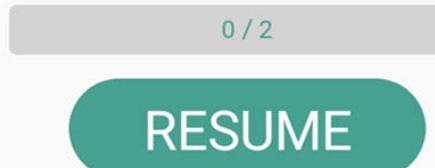




\section{Naming Probe}

The user is first presented with the target item and asked to name it.

The naming probe is presented two more times for each item: 1) following presentation of all of the semantic feature or phonological component cues; 2) following a summary review of cues presented.

The red 'wave' icon indicates that the app is recording the participant's response.

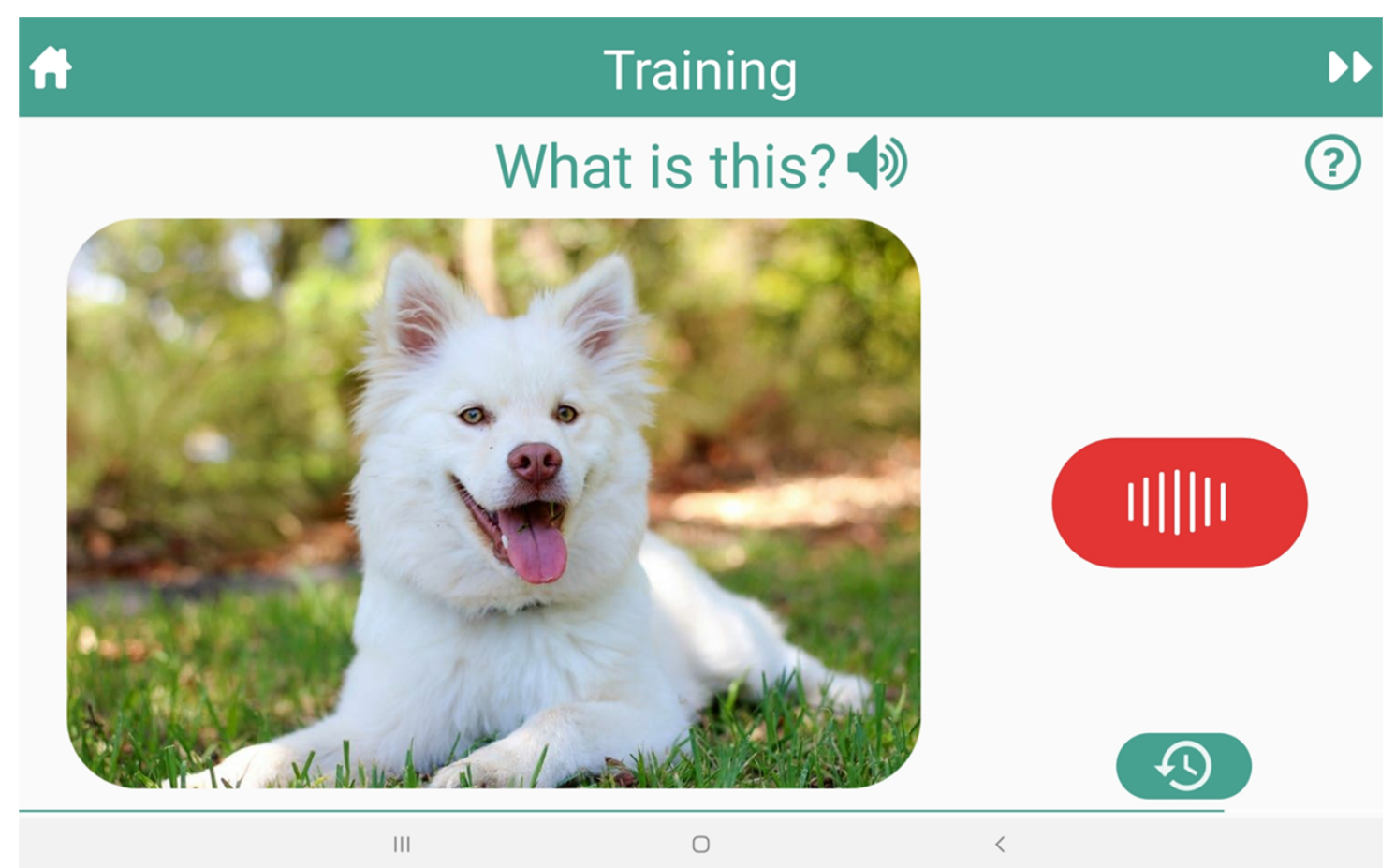




\section{Semantic Feature Analysis (SFA)}

Following the initial naming probe, the app presents the following cues:

- Category or group: "What category or group does this item belong to?"

- Association: "What does this make you think of?"

- Action: "What is it used for?"

- Properties: "What properties does the item have?"

- Location: "Where can you find the item?"

A second probe ("Now try to name the picture again. What is this?") is presented, followed by a Summary page reviewing all of the cues and one final probe ("Name the picture one last time.")

The following slide shows a screenshot example of one of the SFA prompts presented to users of the app. 


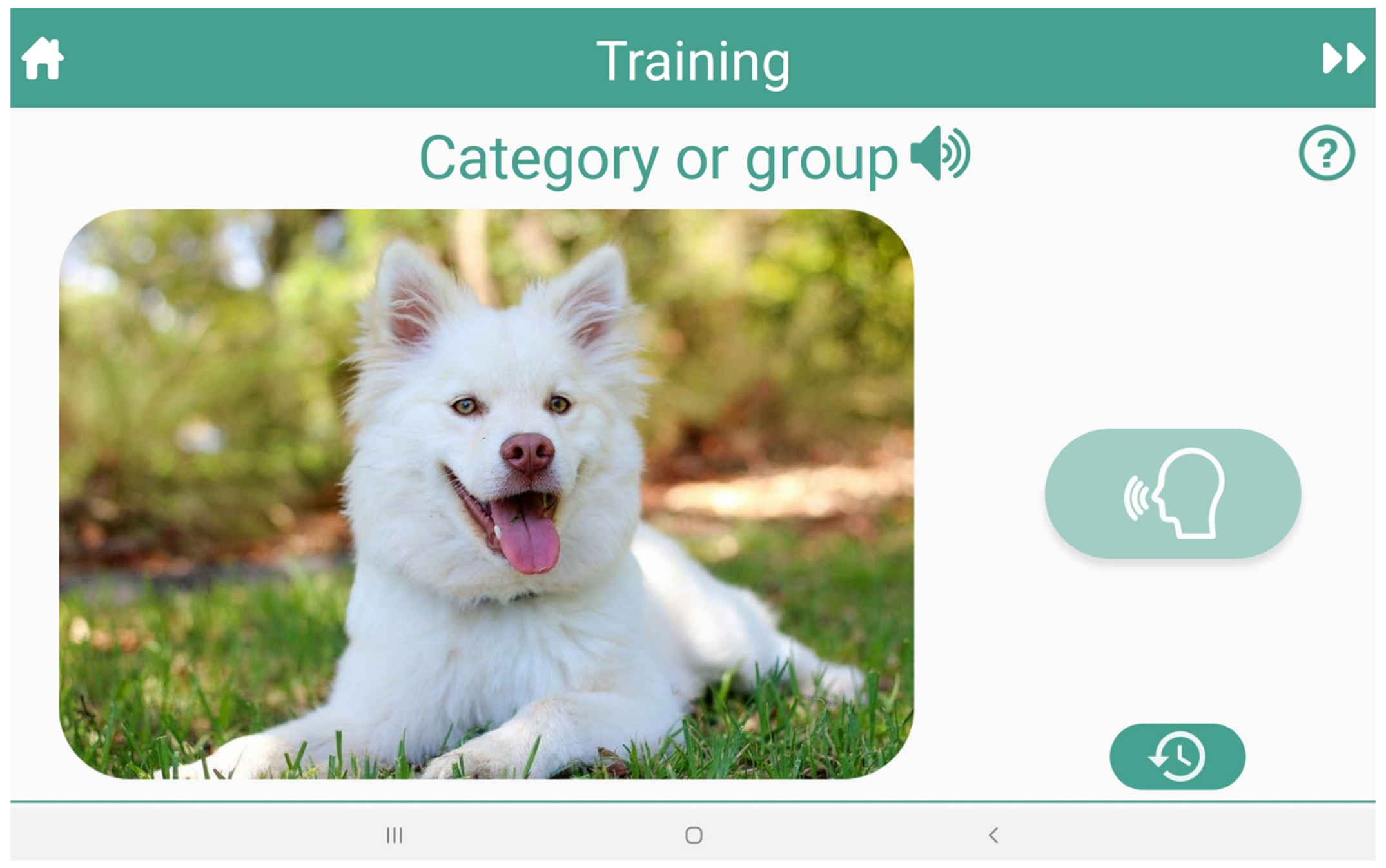




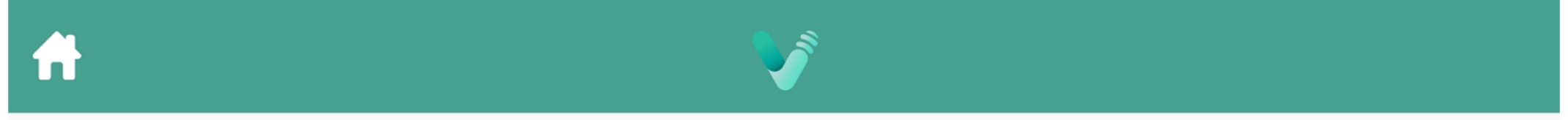

\section{Summary :}

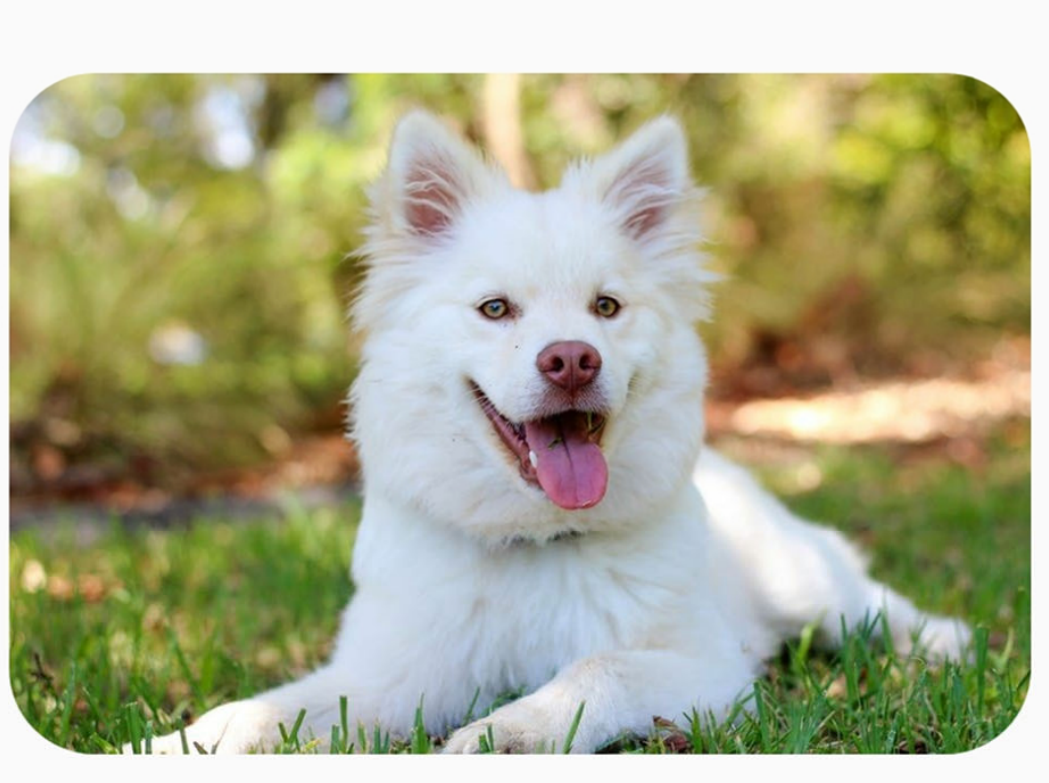

1. Category or group : ANIMALS

2. Association : a wagging tail

3. Action : bark

4. Properties : has four paws

5. Location : in a kennel 


\section{Phonological Components Analysis (PCA)}

Following the initial naming probe, the app presents the following cues:

- Rhyme: "What rhymes with the word?"

- First sound: "What sound does the word in the picture start with?"

- Other word: "What is another word with the same first sound?"

- Last sound: "What is the last sound in this word?"

- Number of syllables: "How many syllables does the word have?"

A second probe ("Now try to name the picture again. What is this?") is presented, followed by a Summary page reviewing all of the cues and one final probe ("Name the picture one last time.")

The following slide shows a screenshot example of one of the PCA prompts presented to users of the app. 


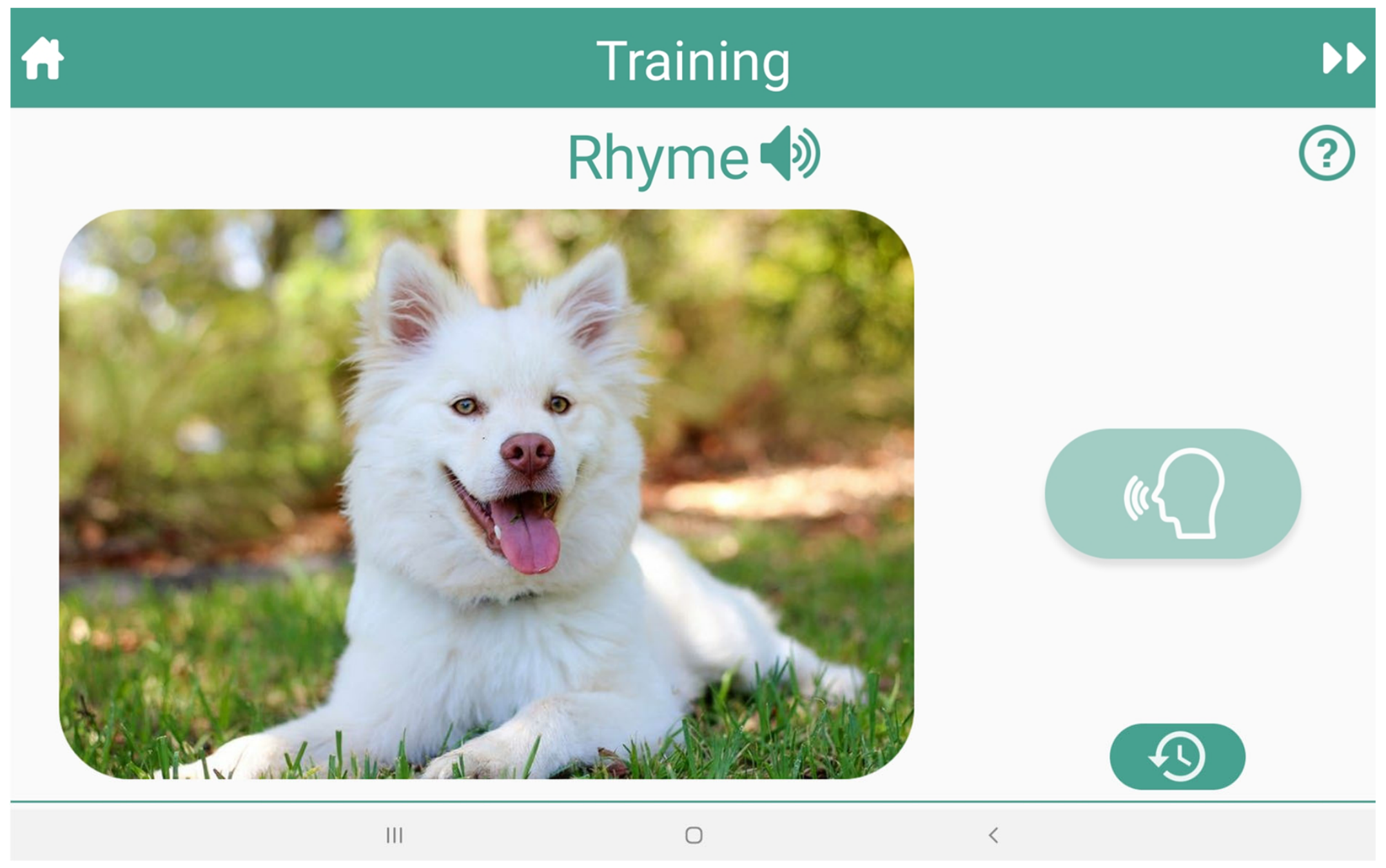




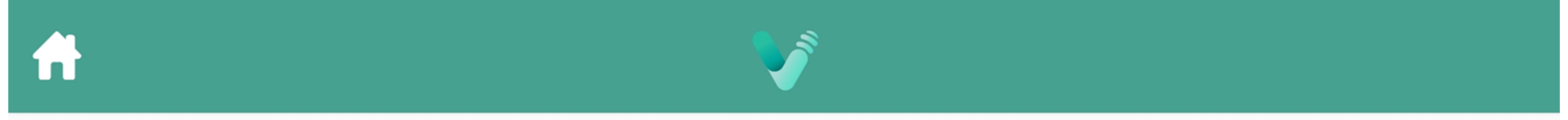

\section{Summary :}

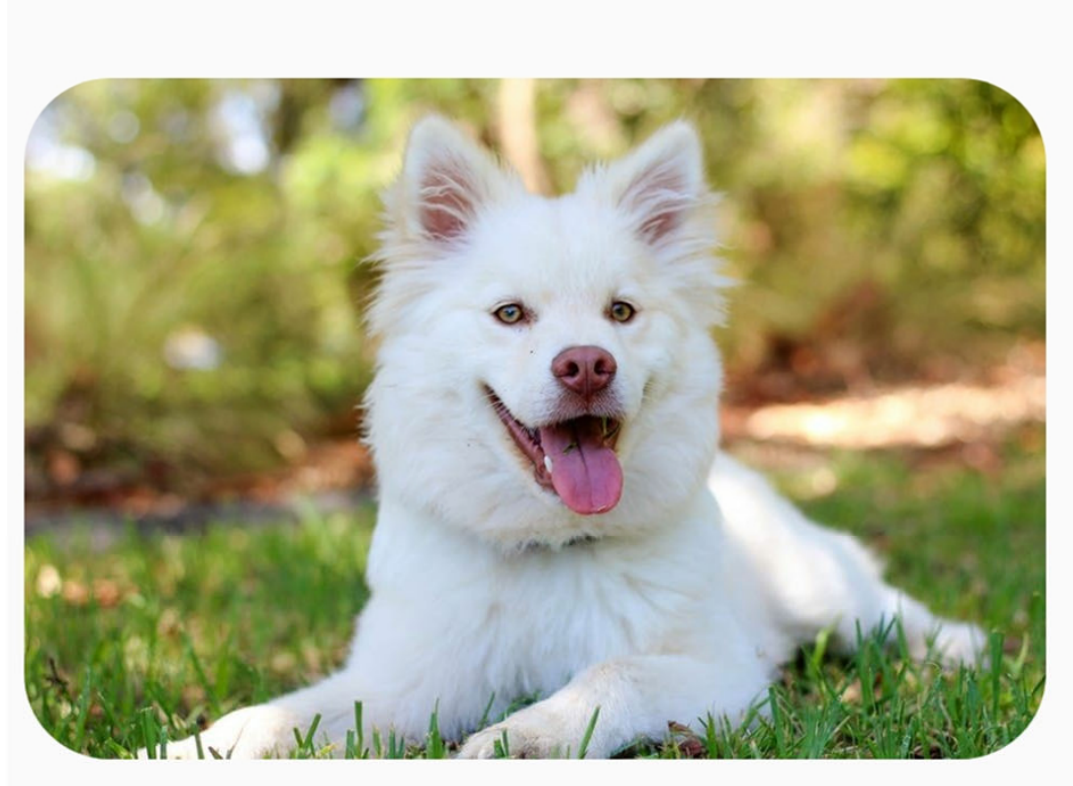

1. Rhyme: fog

2. First sound : $d$

3. Other word : diamond

4. Last sound: $g$

5. Number of syllables : 1 


\section{Final Screen}

The user is presented with a final feedback screen before progressing to the next item.

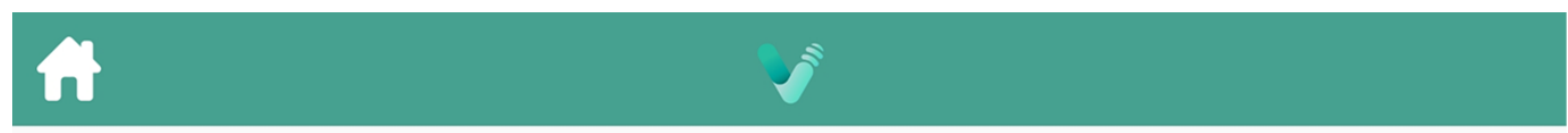

\section{Great, carlee!}

You finished the word "dog". Let's continue with the next word.
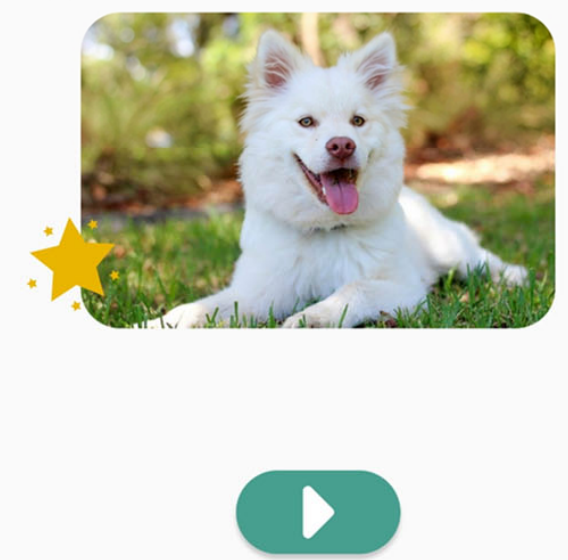

III 